\title{
DISTRIBUCIONES DE ARTEFACTOS LÍTICOS, CRONOLOGÍA Y EL MODELO DE PISOTEO (BORRERO 1988): LOS MÉDANOS DE LA COSTA NORTE DEL LAGO VIEDMA (SANTA CRUZ, PATAGONIA ARGENTINA) ${ }^{1}$
}

\author{
LITHIC ARTIFACT DISTRIBUTIONS, CHRONOLOGY, AND THE TRAMPLING \\ MODEL (BORRERO 1988): SAND DUNES ON THE NORTH SHORE OF LAKE \\ VIEDMA (SANTA CRUZ, ARGENTINE PATAGONIA)
}

\author{
Juan Bautista Belardi ${ }^{2}$, Flavia Carballo Marina ${ }^{2}$ y Patricia Campan ${ }^{2,3}$
}

\begin{abstract}
Luis Alberto Borrero cambió la forma en que se generaba conocimiento arqueológico en la Patagonia y Tierra del Fuego (y más allá también). La interrelación entre la arqueología distribucional y la tafonomía regional -dos programas de investigación introducidos por él hace 30 años- resalta la importancia de tres unidades analíticas: la región, el artefacto y el elemento (óseo). Esta es la estructura, junto con una epistemología falsacionista, para formular preguntas amplias relacionadas principalmente con el uso del paisaje por parte de poblaciones cazadoras recolectoras a lo largo del Holoceno. Sobre la base de este legado se presenta un caso de estudio de los campos de invernada de la margen norte del Lago Viedma. Se integran los resultados de las distribuciones de artefactos con los de la tafonomía de guanacos (Lama guanicoe) y se dan a conocer nuevas cronologías radiocarbónicas que enmarcan el análisis de la fauna de sitios arqueológicos ubicados en hoyadas de deflación en médanos de la costa. La identificación de condiciones ambientales y arqueológicas semejantes a las propuestas por Borrero en 1988 en el Modelo de Pisoteo de guanacos para Tierra del Fuego, muestra la relevancia para su aplicación a la región del lago Viedma.
\end{abstract}

Palabras claves: Lago Viedma, distribuciones artefactuales, cronología, Modelo de Pisoteo, guanaco.

Luis Alberto Borrero changed the way of producing archaeological knowledge in Patagonia and Tierra del Fuego (as well as beyond). The interrelationship between distributional archaeology and regional taphonomy-two research programs introduced by him 30 years ago-highlights the importance of three analytical units: the region, the artifact and the element (bone). Combined with an epistemology centered on falsifiability, this is the structure for formulating broad questions mostly related to Holocene hunter-gatherer landscape use. Building on this legacy, the article presents a case study of the winter grounds on the north shore of Lake Viedma. It integrates the taphonomic results of guanaco (Lama guanicoe) with those of artifact distribution. Furthermore, we present new radiocarbon dates that frame the analysis of fauna from several archaeological sites located in the deflation hollows of coastal sand dunes. The identification of a similar set of environmental and archaeological conditions to that proposed by Borrero in 1988 in the trampling model he used for guanacos in Tierra del Fuego demonstrates the latter's relevance to the Viedma Lake region.

Key words: Lake Viedma, artifact distributions, chronology, trampling model, guanaco.

El Dr. Luis A. Borrero, a lo largo de su amplia trayectoria profesional, ha tenido (y tiene) una influencia en la arqueología de Patagonia y Tierra del Fuego que impactó en la formación de generaciones de arqueólogos y, consecuentemente, en el desarrollo de proyectos de investigación llevados a cabo en muy diferentes ecosistemas y con problemáticas muy variadas. Uno de los aspectos centrales de la influencia

1 Una primera versión de este trabajo fue presentada en el Simposio Patagonian evolutionary archaeology and human paleoecology: Commending the legacy (still in the making) of Luis Alberto Borrero in the interpretation of hunter-gatherer studies of the Southern Cone, realizado en el marco de la reunión anual de la Society for American Archaeology realizada en Albuquerque, USA (abril 2019). Este manuscrito fue revisado por pares externos y editado por el Comité Editor de Chungara y por uno de los editores invitados, César Méndez.

2 Universidad Nacional de la Patagonia Austral, Unidad Académica Río Gallegos (ICASUR), Laboratorio de Arqueología Dr. Luis A. Borrero, CONICET, Campus Universitario, Río Gallegos, Argentina. juanbautistabelardi@gmail.com

3 Secretaría de Estado de Cultura de la Provincia de Santa Cruz, Rio Gallegos, Argentina. pxcampan@gmail.com 
de Borrero es que las preguntas que guían dichos proyectos se orientan desde la ecología evolutiva, donde las poblaciones humanas son entendidas como un componente más del ecosistema. Además, permite evaluar tanto el corto como el largo plazo desde una perspectiva biogeográfica e interdisciplinaria que conlleva una escala espacial regional (Borrero 1993) y una epistemología centralizada en la falsación (Popper 1980).

De todos los aportes plasmados por Borrero y con el fin de mostrar la relevancia de la generación de información en grandes espacios, se seleccionaron aquellos relacionados con la arqueología distribucional (Borrero et al. 1992) y con la tafonomía regional (Borrero 1988a y b). Los resultados de la aplicación de la interrelación entre ambos enfoques se exponen tomando como caso de estudio los médanos de la costa de la margen norte del lago Viedma, en el suroeste de provincia de Santa Cruz (Argentina).

Luis Borrero transformó la arqueología de la Patagonia y Tierra del Fuego al introducir en los diseños de sus investigaciones los estudios distribucionales pioneros llevados a cabo por David H. Thomas (1975) en el valle Monitor, California, y por Robert Foley (1981) en Amboseli, Kenia. Esto implicó trasladar la unidad de análisis espacial desde el sitio a la región. Si bien, a comienzos de la década de 1980, Carlos Aschero y su equipo aplicaron un enfoque regional semejante en Piedra Parada (centro de la provincia del Chubut), la principal diferencia entre ambos acercamientos es que Borrero tuvo en cuenta todo el registro arqueológico -ya sea que se encuentre aislado y/o concentrado- incluyendo los procesos de formación y no solamente los sitios.

La arqueología distribucional considera que el registro arqueológico tiene una manifestación espacial relativamente continua con variaciones en su densidad (Borrero et al. 1992; Dunnell y Dancey 1983; Foley 1981, entre otros). Esto se sustenta en que la captura de energía del ambiente y el comportamiento humano son espacialmente continuos. La perspectiva del paisaje resulta conveniente para encuadrar estudios distribucionales, ya que permite abordar un espacio heterogéneo y presupone, además, una jerarquización del mismo por parte de las poblaciones humanas establecida por el conocimiento de diferencias tanto en la oferta de recursos como ideacionales. Así, un objetivo es reconocer los paisajes arqueológicos, entendidos como el resultado de la sumatoria y distribución del total de restos arqueológicos a lo largo del tiempo en un espacio dado junto con sus procesos de formación
(Belardi y Borrero 1999; Binford 1987; Borrero et al. 1992; Rossignol y Wandsnider 1992). El espacio es considerado como un recurso que condiciona la forma en que los demás se distribuyen (Binford 1989); en este sentido no es un mero escenario sino el resultado de procesos coevolutivos. Las poblaciones humanas integran y participan de los ecosistemas modificándolos y siendo, a su vez, afectadas por ellos. Así, el ambiente se convierte en una variable dinámica (Winterhalder y Smith 1992). El conocimiento que las poblaciones cazadoras recolectoras tienen de su entorno conlleva la noción de paisajes heredados, donde sus modificaciones se trasmiten de generación en generación como una forma de herencia ecológica (construcción de nicho -Laland y O'Brien 2010). Desde esta perspectiva el concepto de variabilidad es central para considerar al registro arqueológico y a sus procesos de formación y, a la vez, favorece la comparación e integración de la información arqueológica y paleoambiental.

La introducción del enfoque distribucional fue acompañado por el desarrollo de la tafonomía regional que enfatiza "...el estudio de las distribuciones de animales muertos y sus posteriores historias tafonómicas dentro de un espacio más grande. Se trata de reconocer aquellos espacios dentro de los que hay mayores posibilidades para la depositación, enterramiento y preservación de huesos" (Borrero 1988a:9). A la vez, llama a la identificación del rango de procesos potenciales que permiten establecer un control específico sobre factores que alteran al registro arqueológico. No solo interesa la depositación de esqueletos sino también la de huesos sueltos en consonancia con la de artefactos, ya sea que se presenten agrupados y/o aislados. De esta forma, un objetivo central de la tafonomía regional es mapear zonas de factibilidad de determinadas distorsiones de acuerdo con bloques espacio-temporales (Borrero 1988a).

La combinación de estudios distribucionales con el desarrollo tafonómico regional destaca tres unidades analíticas: la región, el artefacto y el elemento óseo (Borrero 2001a). Su empleo, de manera interrelacionada, se manifiesta en el paisaje arqueológico y pone bajo la misma perspectiva teórica y metodológica a la arqueología y a la tafonomía, al punto que hoy se las piensa de forma indisoluble. El objetivo de este trabajo se focaliza en los médanos de la costa de los campos de invernada ( $\leq 400 \mathrm{msm}$ ) de la margen norte del lago Viedma, sudoeste de la provincia de Santa Cruz. Se relacionan distribuciones de hallazgos aislados, concentraciones artefactuales y de restos faunísticos con sus condiciones de 
depositación, asociación y preservación diferencial a la luz de consideraciones tafonómicas regionales derivadas del Modelo de Pisoteo de guanacos (Lama guanicoe) planteado por Borrero (1988a) para Tierra del Fuego. Para ello, en primer lugar se describen los campos de invernada, el estrés invernal y sus consecuencias sobre las poblaciones de guanacos. Luego, se brinda la información distribucional y se presentan los sitios registrados en hoyadas de deflación en médanos. Se dan a conocer nuevas dataciones radiocarbónicas y se analizan los restos óseos de guanaco. A esto le siguen las consideraciones del Modelo de Pisoteo y la evaluación del potencial de aplicación a los contextos arqueológicos del lago Viedma. Sobre esta base se discute y establece la relación entre los campos de invernada y el estrés sobre las poblaciones de guanaco, el Modelo de Pisoteo y los conjuntos arqueológicos localizados en hoyadas de deflación. Las dataciones anclan cronológicamente las ocupaciones de los médanos en el Holoceno Tardío y el análisis de los restos faunísticos, junto con las consideraciones tafonómicas regionales, permite distinguir un continuum de situaciones de preservación diferencial de restos de guanaco. Así, se amplía el conocimiento de la ocupación humana de la cuenca del Lago Viedma (Belardi y Caracotche 2005; Belardi et al. 2016, 2017, 2019a y b, 2020, 2021; Menghin 1957; Nuevo Delaunay et al. 2020). Además, se constata que los supuestos que subyacen al Modelo de Pisoteo se cumplen a escala suprarregional al identificar, fuera de Tierra del Fuego, condiciones semejantes para su aplicación.

\section{El Lago Viedma: Disponibilidad de Espacios y Estrés Invernal}

El Lago Viedma (78 km de longitud media) es de forma elongada y se orienta en sentido oesteeste como resultado de la dinámica glaciaria del Cuaternario (Figura 1). Sus cabeceras se encuentran conectadas con el Campo de Hielo Patagónico Sur (CHPS), la margen noreste está enmarcada por mesetas basálticas del Mioceno ( $\geq 900 \mathrm{msm}$ ), que hacia el oeste dan lugar a relieves cordilleranos que dificultan la circulación humana hacia el norte. Sus grandes dimensiones favorecen su acción como atemperador de las condiciones climáticas. El oeste corresponde a un ecosistema de bosque de Nothofagus y el este a la estepa graminosa.

Sobre el modelado glaciario han actuado los procesos eólicos construyendo rasgos menores del paisaje regional, tales como los médanos, que se localizan mayoritariamente a lo largo de las costas del lago (Strelin y Malagnino 1996; Wenzens et al. 1997; Pereyra et al. 2002). La datación de niveles del lago indica que los niveles actuales de costa se habrían conformado a partir de $2500 \mathrm{cal} \mathrm{AP}$, como así también los médanos (L. Horta comunicación personal, 2021.). La mayor extensión de estos últimos se asociaría, en particular, a los momentos de marcada aridez que tuvieron lugar durante los últimos 1000 años (Wenzens et al. 1997).

El espacio comprendido entre la costa del lago y la cota de $400 \mathrm{~m}$ es donde se encuentran los cascos de las estancias y, al ser el sector que tiene mayores temperaturas y acumula menor cantidad de nieve (Sturzembaun y Borrelli 2001; Belardi et al. 2019a), es empleado por estos establecimientos ganaderos como campos de invernada (Figura 1). Por esta razón, también concentra poblaciones de guanacos y choiques (Rhea pennata), que se desplazan hacia el lago cuando comienzan a disminuir las temperaturas de las mesetas basálticas -campos de veranada.

El estrés invernal es una característica de la Patagonia y Tierra del Fuego, aunque puede variar en su intensidad. Su efecto decisivo es la mortandad masiva y espacialmente concentrada de animales. Las bajas temperaturas, la cubierta de nieve y la consecuente dificultad de acceso al forraje generan el debilitamiento paulatino de los individuos que, para guardar el calor, tienden a agruparse y a buscar lugares reparados donde mueren. Esto ocurre sobre las poblaciones de guanacos, tanto en abrigos rocosos como en espacios a cielo abierto (ver referencias y discusiones en Rindel y Belardi 2006; Belardi y Rindel 2008; Borrero 2001b, 2009). En el lago Viedma, el lugar de mayor reparo y de menor o nula carga nival es la estrecha franja de médanos y molles (Schinus sp.) (200-300 m) que acompaña segmentos de la línea de costa, por lo que allí tiende a concentrarse la circulación de guanacos.

La conexión de la cuenca con el CHPS determinó que los avances neoglaciares aumentaran las condiciones de estacionalidad habituales hacia el oeste (menores temperaturas y mayor carga nival) de los campos de invernada y que se haya generado mayor aridización (Strelin et al. 2014; Kaplan et al. 2016). Eso se tradujo en condiciones de estrés invernal más rigurosas.

Los trabajos distribucionales se llevaron a cabo a partir de la realización de transectas lineales con muestreos continuos. Los resultados indican una baja 

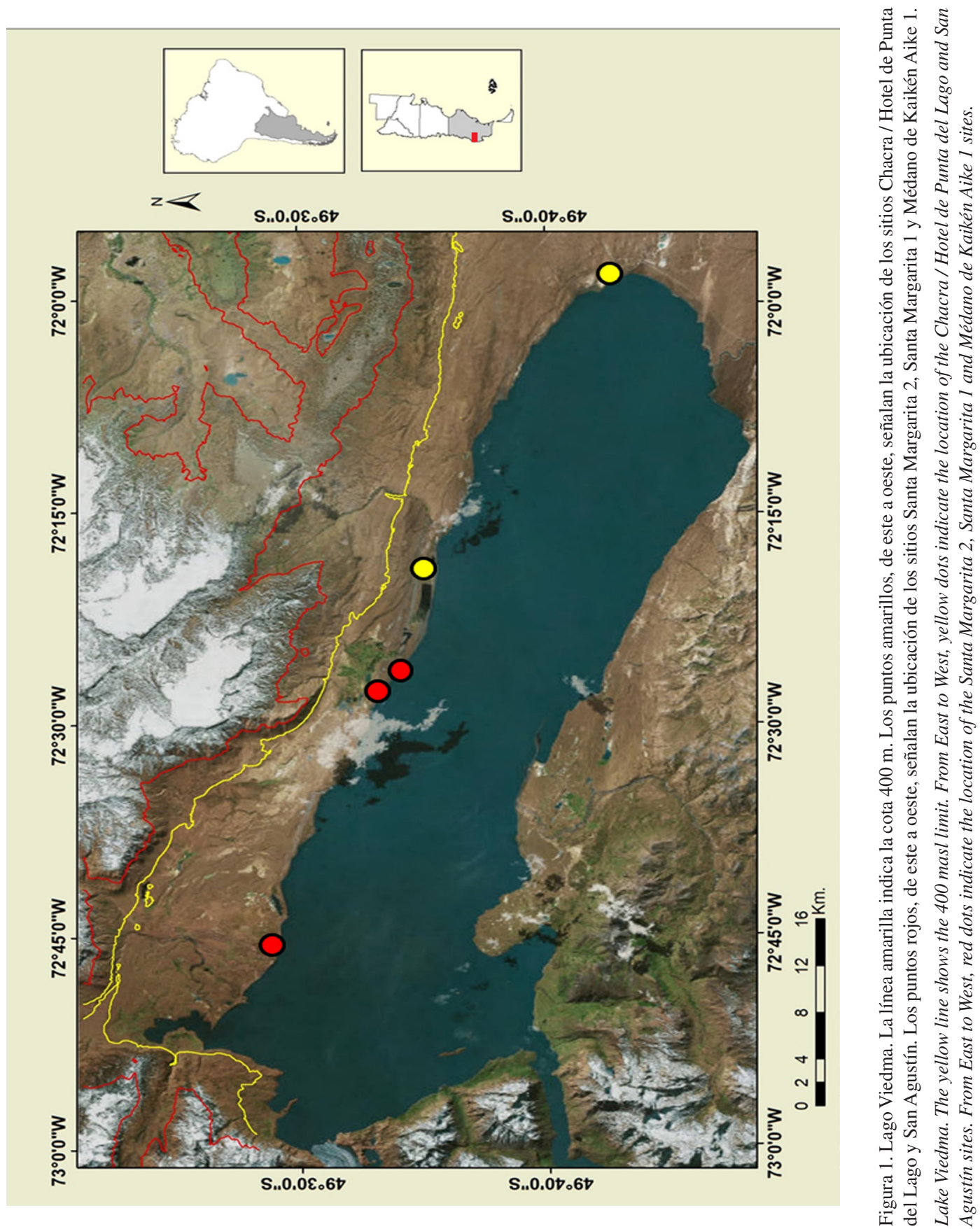
intensidad de uso de la margen norte del lago Viedma por parte de las poblaciones humanas; sin embargo existen diferencias en el paisaje arqueológico entre los distintos niveles altitudinales y entre la estepa y el bosque (Belardi et al. 2020, 2021). Las cronologías radiocarbónicas ubican a las ocupaciones humanas entre los ca. 3700 años AP y el siglo XX, tal es el caso de la reserva Tehuelche-Aonikenk-Mapuche de Cerro Índice (Belardi et al. 2016, 2019b, 2020, 2021; Nuevo Delaunay et al. 2020). No obstante, motivos rupestres pintados del sitio Punta del Lago 1 sugieren una profundidad temporal que se extendería hasta el Holoceno Temprano. Esto tiene sentido dado que en las cuencas de los lagos Tar-San Martín y Argentino, localizadas al norte y al sur respectivamente, las primeras señales de ocupación humana se han datado en torno a los 9700 años AP (Belardi et al. 2019a).

Los campos de invernada son el único espacio que puede ser usado durante todo el año, en especial la costa del lago. Los médanos y los molles ofrecen reparo y leña, y en la playa hay disponibilidad de madera de resaca y rocas aptas para la talla. A ello debe agregarse, como se mencionara, la agrupación de guanacos y choiques durante el invierno. Estas condiciones de refugio estacional, sumadas a la concentración de recursos en un marco regional, darían cuenta del uso planificado por parte de las poblaciones humanas. Al respecto, la costa, luego de las pampas altas (401-900 msm), es el lugar de mayor intensidad de uso, con una densidad artefactual de $600 \cdot 10^{-5}$ $\mathrm{m}^{2}\left(\mathrm{~N}\right.$ artefactual $=263 / 162.000 \mathrm{~m}^{2}$ muestreados $)$ y un $27,10 \%(\mathrm{~N}=107)$ de muestreos con hallazgos. Además, posee la mayor riqueza artefactual (Belardi et al. 2021).

Los puntos con alta frecuencia y riqueza artefactual son discontinuos y se vinculan con materiales líticos $\mathrm{y}$ arqueofaunas de guanaco expuestos en hoyadas de deflación, que se forman ante la ausencia de vegetación; llega a haber más de 1,5 m de diferencia entre el piso de la hoyada y el nivel vegetado de los médanos (Figura 2 A, B y D). Fuera de las hoyadas casi no se recuperaron restos óseos y los pocos que se encuentran presentan estadios de meteorización altos. Esto se explicaría porque, salvo en la línea de médanos, el potencial de entierro es bajo a nulo.

A continuación se presentan los resultados de los sitios Santa Margarita 1 y 2 y Médano de Kaikén Aike 1 (Figuras 1 y 2, Tablas 1 a la 4). El foco está puesto sobre los restos faunísticos de guanaco, que son predominantes. Además, se hace mención a otros conjuntos que, como los sitios Chacra / Hotel de Punta del Lago y San Agustín, solo tienen artefactos líticos (Figura 1). Si bien la información es preliminar, en tanto que los materiales arqueológicos están bajo estudio, permite identificar tendencias y la pertinencia del Modelo de Pisoteo para discutir la arqueología de los médanos. Así, con el fin de ponderar la integridad de los conjuntos óseos se los describe y caracteriza a partir de los valores de NISP (número de especímenes identificados), de acuerdo con sectores anatómicos, sus estadios de meteorización (Beheresmeyer 1978), la presencia de marcas y huellas, y las frecuencias de diáfisis y epífisis de húmero, radiocúbito, fémur y tibia.

Estos sitios se caracterizan por el empleo mayoritario del basalto y la dacita (rocas disponibles en la costa y en las morenas sobre las que apoyan muchos de los médanos) y por la riqueza artefactual. También se registraron artefactos de obsidiana y limolita -en su mayoría desechos-, rocas provenientes de la Pampa del Asador (Espinosa y Goñi 1999) y del Lago Cardiel (Belardi et al. 2015), respectivamente. Además, se recuperaron núcleos y desechos vinculados con actividades de talla, piezas de filos cortos, largos, y molinos y manos. Entre los instrumentos directamente relacionados con la obtención de recursos se destaca el registro de bolas y las evidencias de su manufactura (preformas -poliedros-, lascas y bolas terminadas), seguidas por puntas de proyectil triangulares pedunculadas. La presencia de hojas podría relacionarse con el procesamiento del guanaco. Sobre toda esta evidencia -a la que se suma la faunística que se presenta a continuación- y el uso diferencial de los campos de veranada e invernada por parte de las poblaciones de guanaco y choique, se propuso el empleo residencial y redundante de la costa, tanto sobre base estacional como anual (Belardi et al. 2021).

Los sitios Chacra / Hotel de Punta del Lago y San Agustín (255 msm) se corresponden con muestreos en transectas con alta densidad de hallazgos, en particular en el caso de San Agustín donde se presentan materiales líticos a lo largo de ca. $200 \mathrm{~m}$.

En el sitio Santa Margarita $2(257 \mathrm{msm}$ ) (Figura 2 B-C) se distinguieron dos niveles con restos faunísticos que, si bien contiguos, no se superponen (Tablas 1 a la 4). El primero (denominado "Huesos blancos"), está sobre la superficie de la hoyada de deflación e incluye restos meteorizados en una superficie de $60 \mathrm{~m}^{2}$ y una concentración central de un $\mathrm{m}^{2}$ con fragmentos óseos quemados y calcinados, asociados a una estructura de combustión con rodados tiznados. Entre los restos meteorizados se reconoció un machacador óseo (Hajduk y Lezcano 2005) confeccionado sobre un 


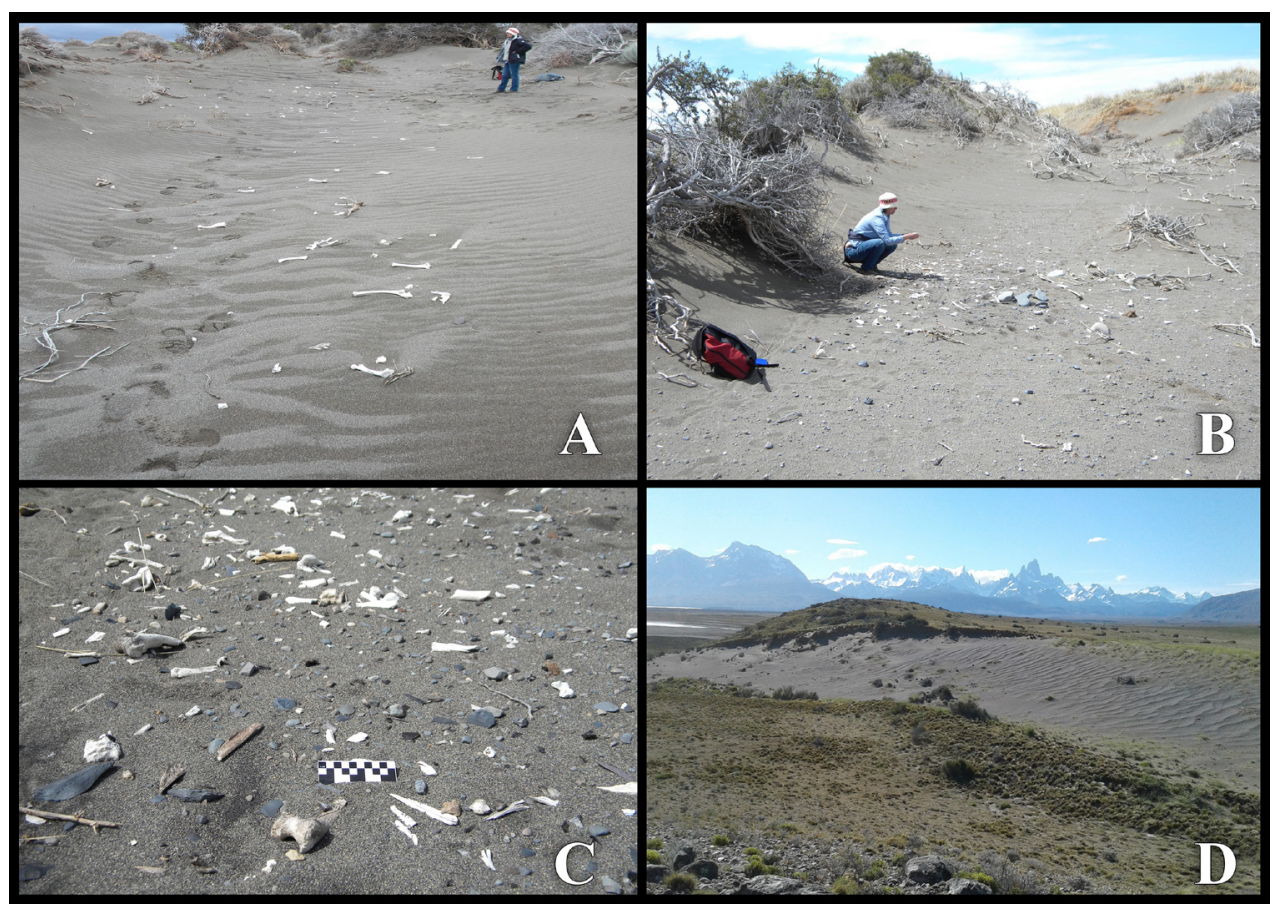

Figura 2. A. Sitio Santa Margarita 1. Los restos óseos de guanaco que se observan en primer plano son modernos. B. Sitio Santa Margarita 2. Sectores norte y central. C. Sitio Santa Margarita 2. Detalle del sector central. Se observan artefactos líticos de basalto y restos óseos en avanzado estado de meteorización. D. Vista de la hoyada de deflación donde se localiza el sitio Médano de Kaikén Aike 1.

A. Santa Margarita 1 site. Present-day guanaco bones in the foreground. B. Santa Margarita 2 site. North and central sectors. C. Santa Margarita 2 site. Detailed view of the central sector. Basalt lithic artifacts and bones at advanced weathering stage can be seen. D. View of deflation hollow where the Médano de Kaikén Aike 1 site is located.

húmero distal derecho de guanaco que fue datado

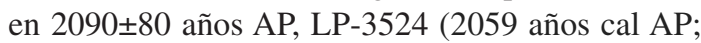
Calib 8.2) (Stuiver et al. 2020).

Unos dos metros hacia el norte, en estratigrafía y cubierto en parte por el médano, se registró el otro nivel de restos óseos. Se lo denominó "Nivel negro" y presenta un buen estado de conservación, aunque los restos se encontraban muy húmedos, con la matriz arenosa impregnada de carbón. Los huesos se recuperaron en dos sondeos lineales: uno de $3 \mathrm{~m}$ por $0,5 \mathrm{~m}$ paralelo al médano y otro de $2 \mathrm{~m}$ por 0,3 $\mathrm{m}$ transversal al mismo. En el sector expuesto, este nivel se encuentra a cinco $\mathrm{cm}$ de la superficie y tiene $10 \mathrm{~cm}$ de potencia. Pruebas de pala señalan que el "Nivel negro" se extiende otros dos metros paralelos al médano y un metro y medio más en sentido transversal (a espaldas de la persona en cuclillas en la Figura 2 B). Se dató otro machacador realizado sobre un húmero distal izquierdo de guanaco en $1790 \pm 80$ años AP, LP-3487 (1683 años cal AP; Calib 8.2) (Stuiver et al. 2020).
Si bien ambas dataciones son muy próximas, las muestras son significativamente diferentes al nivel de significación del 95\% (prueba estadística $\mathrm{T}=7,03125$; Chi cuadrado $(0,5)=3,84 ; 1$ grado de libertad), por lo que se entiende que corresponden a momentos de depositación distintos. Pero, ¿cómo se explica que los restos expuestos en superficie sean más antiguos? Aunque se desconoce la frecuencia de eventos de enterramiento y desenterramiento, dada la dinámica de los médanos, esto pudo suceder repetidas veces (Muñoz 1999). En la Figura 3 se propone un esquema de formación de estos conjuntos (momentos 1-4) que subsume dicha dinámica. Primero se depositó el nivel de "Huesos blancos" (1) y luego el "Nivel negro" (2), que fue tapado por un médano de manera relativamente rápida (3). Dada la datación de los "Huesos blancos", estos también habrían sido tapados -ya que en caso contrario no se conservarían- y se habrían descubierto un tiempo antes del momento de interceptación por parte de este equipo de trabajo (4). En el sitio Santa Margarita 1 (261 msm) (Figura 2 A) se realizó el 
Tabla 1. NISP y porcentajes por región anatómica por sitio / nivel. Referencias: M. d.: miembro delantero, M. t.: miembro trasero. *No se consideraron para el NISP dos machacadores del Nivel "Huesos blancos" (confeccionados sobre húmeros), **tres machacadores del "Nivel negro" (uno sobre húmero y dos sobre fémures) $\mathrm{y} * * * u$ retocador sobre metapodio.

NISP and percentages by anatomical region and site / layer. References: M. d.: hind limb, M. t.: forelimb. *Two crushers from the "Nivel Huesos blancos" (white bones level) (made on humeri), **three crushers from the "Nivel negro" (black Level) (one made on a humerus and two on femurs) and ***one retoucher made on a metapodial were not considered for the NISP.

\begin{tabular}{lllll}
\hline \multicolumn{5}{c}{ Sitios / nivel } \\
\hline $\begin{array}{l}\text { Segmento anatómico } \\
\text { Santa Margarita 1 }\end{array}$ & Santa Margarita 1 & $\begin{array}{l}\text { Santa Margarita 2 } \\
\text { Superficie } \\
\text { "Huesos blancos" }\end{array}$ & $\begin{array}{l}\text { Santa Margarita 2 } \\
\text { Estratigrafía } \\
\text { "Nivel negro" }\end{array}$ & $\begin{array}{l}\text { Médano de } \\
\text { Kaikén Aike }\end{array}$ \\
\hline Cabeza & $1(1,13 \%)$ & 0 & $6(6,89 \%)$ & $5(7,24 \%)$ \\
\hline Axial & $11(12,5 \%)$ & 0 & $26(29,88 \%)$ & $20(28,98 \%)$ \\
\hline M. d. superior* & $12(13,63 \%)$ & $16(20 \%)$ & $3(3,44 \%)$ & $16(23,18 \%)$ \\
\hline M. d. inferior & $17(19,31 \%)$ & $24(30 \%)$ & $19(21,83 \%)$ & $10(14,49 \%)$ \\
\hline M. t. superior** & $10(11,36 \%)$ & $17(21,25 \%)$ & $4(4,59 \%)$ & $7(10,14 \%)$ \\
\hline M. t. inferior & $12(13,63 \%)$ & $5(6,25 \%)$ & $3(3,44 \%)$ & $5(7,24 \%)$ \\
\hline Pelvis & $3(3,40 \%)$ & 0 & 0 & 0 \\
\hline Autopodios & $6(6,81 \%)$ & $3(3,75 \%)$ & $12(13,79 \%)$ & $3(4,34 \%)$ \\
\hline Metapodios*** & $9(10,22 \%)$ & $12(15 \%)$ & $7(8,04 \%)$ & 0 \\
\hline Falanges & $7(7,95 \%)$ & $3(3,75 \%)$ & $7(8,04 \%)$ & $3(4,34 \%)$ \\
\hline Total NISP & 88 & 80 & 87 & 69 \\
\hline
\end{tabular}

Tabla 2. Meteorización. NISP y porcentajes por sitio / nivel. Weathering. NISP and percentages by site / layer.

\begin{tabular}{ccccc}
\hline & \multicolumn{4}{c}{ Sitios / nivel } \\
\cline { 2 - 5 } $\begin{array}{l}\text { Estadio } \\
\text { de } \\
\text { meteorización }\end{array}$ & Margarita 1 & $\begin{array}{l}\text { Santa } \\
\text { Margarita 2 } \\
\text { Superficie } \\
\text { "Huesos } \\
\text { blancos" }\end{array}$ & $\begin{array}{l}\text { Manta } \\
\text { Estratigrafía } \\
\text { "Nivel negro" }\end{array}$ & Kaikén Aike 1 \\
\hline 2 & $17(38,63 \%)$ & $16(14,28 \%)$ & $21(95,45 \%)$ & $19(38,77 \%)$ \\
\hline 3 & $22(50 \%)$ & $12(28,57 \%)$ & $1(4,54 \%)$ & $22(44,89 \%)$ \\
\hline 4 & $5(11,36 \%)$ & $14(33,33 \%)$ & 0 & $8(16,32 \%)$ \\
\hline Total NISP & 44 & 42 & 22 & 49 \\
\hline
\end{tabular}

Tabla 4. NISP de epífisis y diáfisis de huesos largos (húmero, radiocúbito, fémur y tibia) por sitio / nivel.

NISP of long bone diaphyses and epiphyses (humerus, radiocubitus, femur, tibia) by site / layer.

\begin{tabular}{cclcc}
\hline \multirow{2}{*}{$\begin{array}{c}\text { Segmentos } \\
\text { anatómicos }\end{array}$} & \multicolumn{4}{c}{ Sitios / nivel } \\
\cline { 2 - 5 } & $\begin{array}{l}\text { Santa } \\
\text { Margarita 1 }\end{array}$ & $\begin{array}{l}\text { Margarita 2 } \\
\text { "Huesos blancos" "Nivel negro" }\end{array}$ & $\begin{array}{l}\text { Santa } \\
\text { Margarita 2 }\end{array}$ & $\begin{array}{l}\text { Kédano de } \\
\text { Kaikén Aike 1 }\end{array}$ \\
\hline Epífisis & 37 & 41 & 12 & 25 \\
\hline Diáfisis & 11 & 19 & 17 & 10 \\
\hline
\end{tabular}

Tabla 3. Marcas y huellas. NISP y porcentajes por sitio / nivel. Referencias: $\mathrm{n} / \mathrm{d}$ : $\sin$ datos.

Natural and cultural marks. NISP and percentages by site / layer. References: $n / d$ : no data.

\begin{tabular}{|c|c|c|c|c|}
\hline \multirow[b]{2}{*}{ Marcas y huellas } & \multicolumn{4}{|c|}{ Sitios / nivel } \\
\hline & $\begin{array}{l}\text { Santa } \\
\text { Margarita } 1\end{array}$ & $\begin{array}{l}\text { Santa } \\
\text { Margarita } 2 \\
\text { Superficie } \\
\text { "Huesos } \\
\text { blancos" }\end{array}$ & $\begin{array}{l}\text { Santa } \\
\text { Margarita } 2 \\
\text { Estratigrafía } \\
\text { "Nivel negro" }\end{array}$ & $\begin{array}{l}\text { Médano de } \\
\text { Kaikén Aike } 1\end{array}$ \\
\hline Marcas raíces & $40(76,92 \%)$ & $3(100 \%)$ & $58(69,87 \%)$ & $36(83,72 \%)$ \\
\hline Marcas roedor & $4(7,69 \%)$ & 0 & $4(4,81 \%)$ & 0 \\
\hline Abrasión hídrica & 0 & 0 & $17(20,48 \%)$ & $1(2,32 \%)$ \\
\hline Termoalteración & $5(9,61 \%)$ & $\mathrm{n} / \mathrm{d}$ & $1(1,20 \%)$ & $4(9,30 \%)$ \\
\hline Huellas de corte & $1(1,92 \%)$ & 0 & 0 & 0 \\
\hline $\begin{array}{l}\text { Fractura } \\
\text { longitudinal }\end{array}$ & $2(3,84 \%)$ & 0 & $1(1,20 \%)$ & 0 \\
\hline Huellas de golpe & 0 & 0 & $2(2,40 \%)$ & $2(4,65 \%)$ \\
\hline Total NISP & 52 & 3 & 83 & 43 \\
\hline
\end{tabular}




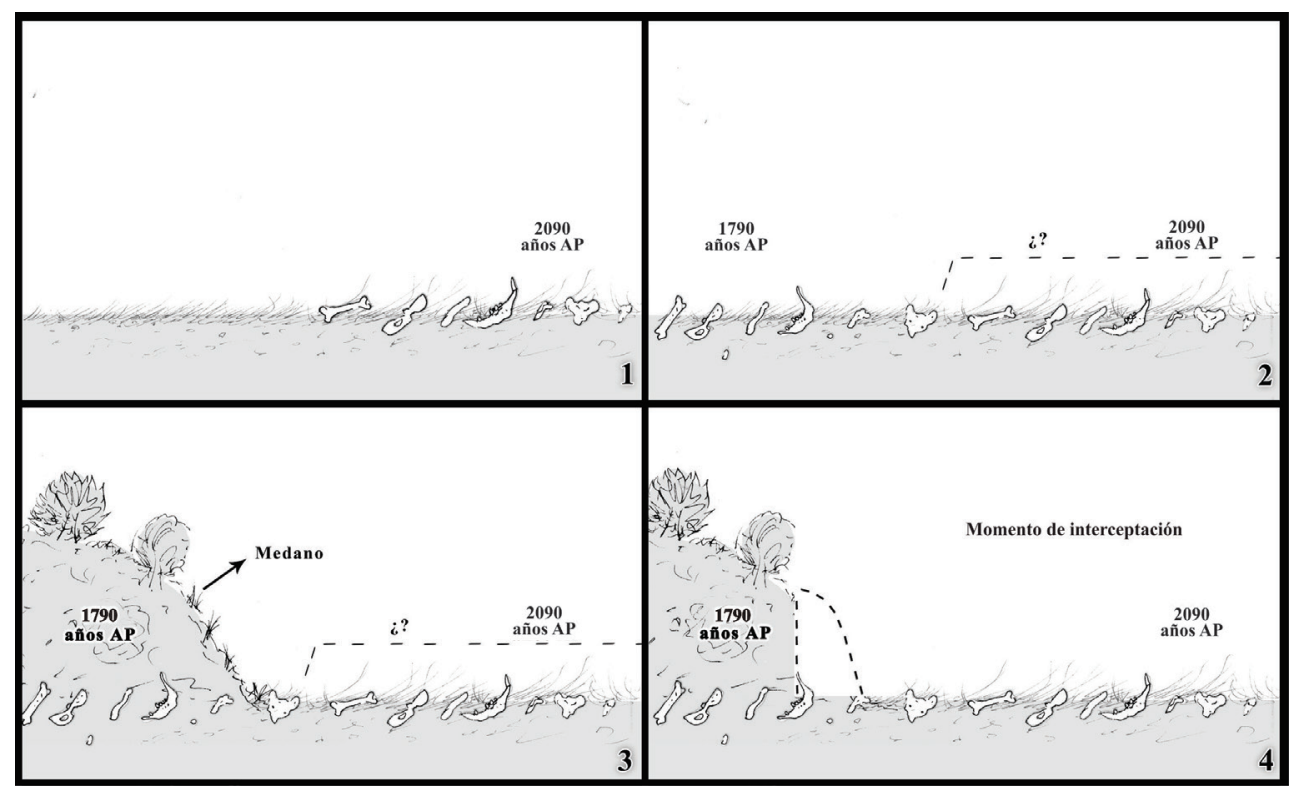

Figura 3. Esquema de formación del sitio Santa Margarita 2.

Formation scheme for the Santa Margarita 2 site.

relevamiento y recolección de materiales líticos y óseos en una superficie de $50 \mathrm{~m}^{2}$. No se registraron materiales en estratigrafía. Se dató una hemipelvis izquierda con huellas de corte $\left(\mathrm{CO}_{2}\right.$ extraído por el LATyR) en $987 \pm 36$ años AP, AA-113567 (953 años cal AP; Calib 8.2) (Stuiver et al. 2020).

El sitio Médano de Kaikén Aike 1 (271 msm) es el de mayor extensión, lo que se condice con las grandes dimensiones de la hoyada (Figura 2 D). Los materiales se distribuyen en una superficie de al menos $120 \mathrm{~m}(\mathrm{E}-\mathrm{O})$ por $25 \mathrm{~m}$ (N-S). Se reconocieron nueve concentraciones -tanto de artefactos líticos como de fauna- que se recogieron de acuerdo con su distribución espacial en superficie en cuadrículas de dimensiones variables. Sobre el perfil del médano que enmarca la hoyada por el lado norte se recuperó un radiocúbito izquierdo de guanaco con dos negativos de golpes (en uno se conservaba la lasca que no había terminado de desprenderse), que fue datado en 1964 \pm 32 años AP, AA114987 (1893 años cal AP; Calib 8.2) (Stuiver et al. 2020) (comunicación personal L. Horta, 2021).

Los perfiles de regiones anatómicas de los distintos sitios son relativamente similares: predomina el esqueleto apendicular sobre el axial (Tabla 1), lo que es coincidente con el patrón observado por De Nigris y Mengoni Goñalons (2004) en sitios de Patagonia. Se debe notar que el nivel "Huesos blancos" no tiene elementos axiales. La meteorización ha impactado decididamente sobre todos los conjuntos, aunque en menor grado en el "Nivel negro", que tiene el mayor porcentaje de sus restos en estadio 2 (Tabla 2). Debe considerarse que el sitio Santa Margarita 1 tiene mil años menos que los demás conjuntos, por lo que su grado de exposición habría sido mayor. Dados estos perfiles de meteorización, es muy probable que las muestras estén sesgadas por la destrucción de elementos, sobre todo en los conjuntos de superficie. Sin embargo, Médano de Kaikén Aike 1 es el sitio con mayor representación de elementos axiales, que son los de menor densidad mineral ósea (Elkin 1995) y, por lo tanto, los de menores posibilidades de preservación. Esto puede ser visto como un alerta ante una eventual contaminación por huesos modernos. El dinamismo del ambiente se manifiesta en la frecuencia de marcas de raíces (Tabla 3), lo que indica que en algún momento estuvieron enterrados, tal es el caso del "Nivel negro". La baja frecuencia de estas marcas en el nivel "Huesos blancos" podría asociarse con un mayor tiempo de exposición de los restos, con el consecuente predominio de la meteorización obliterando marcas y huellas. En función de la discusión brindada por este trabajo, se acota que en ninguno de los sitios se registraron marcas de pisoteo. De la misma manera, es baja la representación de marcas de roedor y termoalteración. No obstante, respecto de esta última variable, resta analizar el caso del 
sitio Santa Margarita 2 "Huesos blancos", donde, tal como se mencionara, sí se observó una importante frecuencia de fragmentos óseos vinculada con un fogón. El único porcentaje significativo de abrasión hídrica corresponde al "Nivel negro", lo que podría deberse a la mayor humedad de la matriz (Tabla 3). En todos los conjuntos también hay una marcada preservación diferencial de las epífisis por sobre las diáfisis (Tabla 4), lo que resulta contrario a las expectativas derivadas de sus valores de densidad mineral ósea. Esta situación se condice con la observada en otros contextos de médanos del sur de Patagonia (tanto arqueológicos como tafonómicos), donde se propuso que el patrón identificado sería el resultado de la meteorización y la abrasión actuando de forma diferencial sobre los tipos y la posición de los tejidos (fibras de colágeno) de las diáfisis y las epífisis. A ello se agrega la incidencia de la radiación solar que elimina la humedad de los huesos, en primer lugar de las diáfisis. Este proceso se aceleraría en contextos antrópicos donde los huesos se fracturan para la obtención de nutrientes (Belardi et al. 2012). Es interesante notar que lo mismo sucede en el "Nivel negro". ¿Es posible entonces que el tiempo que medió entre su depositación y enterramiento haya sido suficiente para generar dicha representación diferencial? Aunque no se puede brindar una respuesta cabal, se considera que el tiempo sí fue suficiente para que los restos adquirieran el estadio 2 de meteorización.

El conjunto óseo del "Nivel negro", con menores valores de meteorización, mayor frecuencia de marcas de raíces -coincidentes con su situación en estratigrafía- y huellas de golpe, es el de mayor integridad. En el resto de los conjuntos son muy acotadas las inferencias que se pueden realizar sobre el procuramiento / procesamiento / consumo, más allá de haber podido identificar un componente antrópico en su formación.

\section{El Modelo de Pisoteo y su Pertinencia para la Arqueología del Lago Viedma}

Necesitamos muchos sitios, en una zona donde se espere stress invernal sobre las poblaciones animales, para tener un importante problema de migración vertical de huesos. ...Pero ocurre que las condiciones establecidas no son suficientes. Hay que incorporar, por ejemplo, características del substrato (Borrero 1988a:11).
En su trabajo "Tafonomía regional", Borrero (1988a) presenta el modelo del pisoteo de guanacos, el cual fue formulado a partir de observaciones realizadas en cabo San Pablo, Tierra del Fuego, sobre la base del registro de la contaminación recurrente de sitios con huesos de guanacos modernos. El tránsito de los guanacos de forma repetida por los mismos senderos en zonas con una alta frecuencia de sitios, aumenta las posibilidades de que un animal muera sobre uno de ellos por estrés invernal y que sea luego pisoteado por los demás. En sustratos blandos, dicha acción facilita el enterramiento de huesos y la consecuente contaminación de contextos arqueológicos por huesos modernos, lo que trae aparejado el problema metodológico de que se yerre en la elección de las muestras para datarlo. Así, las evaluaciones de casos a la luz del modelo se orientan a evitar interpretaciones lineales del registro arqueológico y al reconocimiento de situaciones de integridad diferencial (Borrero 1988a).

Las tres condiciones establecidas por el Modelo de Pisoteo (alta densidad de sitios, estrés invernal y sustrato que favorece el enterramiento) se cumplen en los médanos de la costa de la margen norte del Lago Viedma. Los trabajos arqueológicos mostraron que, dentro de los campos de invernada, son los médanos los que presentan la mayor densidad artefactual y concentraciones de restos faunísticos, que en casos como los presentados pueden ser definidos como sitios. Como se mencionara, los materiales se encuentran sobre arena suelta en las hoyadas de deflación, lo que favorece el rápido enterramiento de cualquier elemento que reciba presión desde arriba, por ejemplo, al ser pisado.

Durante los trabajos de campo en noviembre de 2016 se registraron los restos desarticulados de un guanaco deslizándose por la pendiente este del médano que enmarca al sitio Santa Margarita 1 (Figura 2 A). A la vez, en una hoyada de deflación ubicada a 75 $\mathrm{m}$ al sur del sitio Santa Margarita 2, se registró un conjunto de restos óseos de guanaco (Figura 4 A-D) (se desconoce el año de su formación). Corresponden al menos a 15 individuos (estimado sobre la base de cráneos), tanto juveniles como adultos, que se localizan sobre la ladera norte de un médano, que es la que recibe mayor insolación. En la figura se ve como los huesos se están desplazando pendiente abajo, quedando entrampados en la vegetación. Esto puede deberse a que los guanacos buscan ganar altura ante el aumento de la cobertura nival, y mueren en la parte alta del médano (situación que podría ser similar al caso del guanaco del sitio Santa Margarita 1). A la 




Figura 4. Guanacos muertos por estrés invernal. A-D: diferentes perspectivas de la dispersión de restos óseos. Nótense tanto elementos aislados enteros y fracturados (huesos largos, vértebras y pelvis) y restos articulados (conjunto de vértebras cervicales, toráxicas y cráneos y mandíbulas).

Guanacos killed by winter stress. A-D: A-D: different views of the bone dispersion. Note both the whole and fractured bones (long bones, vertebrae, and innominate bones) along with jointed bones (cervical and thoraxic vertebrae sets, skulls, and mandibles).

vez, el conjunto reúne las características de muerte por estrés invernal: alta frecuencia de individuos en un espacio acotado (Figura 4 A-D), superposición de restos (Figura 4 B-D), presencia de carcasas enteras (en este caso se encuentran ya mayormente desarticuladas), alta frecuencia de elementos axiales articulados (en especial vértebras) (Figura 4D), alta proporción de elementos enteros (Figura $4 \mathrm{D}$ ), una representación variada de sexos y grupos etarios y perfiles de meteorización similares entre individuos (Figura 4 D). No obstante, puede haber diferencias entre individuos superpuestos respecto de la incidencia de la meteorización (Belardi y Rindel 2008). Un caso con características similares a las recién enumeradas fue descrito por Ch. Darwin en las orillas del Río Santa Cruz (ver Borrero 2009).

Borrero (1988b) realizó tres consideraciones más con respecto al pisoteo. La primera se relaciona con su accionar como proceso desarticulador de carcasas, que se puede hacer extensivo a la dispersión de conjuntos óseos en general. La segunda tiene que ver con la fractura de huesos, especialmente de aquellos que son planos (escápula y pelvis) o muy obstrusivos (cráneo y vértebras) (Borrero 1988b). La última, se relaciona con la ausencia de marcas. Las dos consideraciones finales son además relevantes cuando los sustratos son blandos, como en los médanos.

\section{Discusión y Conclusiones}

La información distribucional de los campos de invernada presenta la señal arqueológica más fuerte en los médanos de la costa por su densidad y riqueza artefactual lítica y por la presencia de restos óseos. Esto indica redundancia genérica y un uso más intenso de la costa respecto del interior inmediato por parte de las poblaciones cazadoras recolectoras. Dicha intensidad de uso es solo comparable con la registrada en los campos altos de veranada y, en especial, en la meseta del Tobiano, vinculada con actividades de caza 
de guanaco y también residenciales. De esta forma, existe complementariedad funcional establecida sobre una base ecológica (temperaturas y disponibilidad de recursos - guanacos y choiques) entre los campos de invernada y los de veranada (Belardi et al. 2021). Las tres nuevas dataciones radiocarbónicas de los sitios en médanos Santa Margarita 1 y 2 (ca. 2100-1000 AP) se inscriben en el marco cronológico regional y se ajustan a la establecida sobre base geomorfológica.

Las concentraciones de restos óseos de guanaco, asignados a eventos de mortandad por estrés invernal en torno a las hoyadas de deflación, indican una importante y relativamente constante lluvia natural de huesos. Al ser la costa el lugar de refugio de las poblaciones de guanacos y estar confinado el espacio para circular, los huesos pueden integrarse a los contextos arqueológicos por acción del pisoteo o por la simple mezcla con sitios expuestos, situación usual en las hoyadas de deflación. Además, el pisoteo puede causar descubrimiento, reenterramiento, desplazamientos horizontales y cambiar las condiciones de estabilidad de los restos óseos. Esto fue observado por Borrero (1988b) en cabo San Pablo y por Cruz y Muñoz (2010) en Punta Entrada (costa centro-sur de Santa Cruz). Así, el pisoteo puede tanto mezclar huesos modernos con arqueológicos como contextos arqueológicos. A la vez, aumenta el impacto de la meteorización que, junto con la abrasión, fracturan los restos óseos y facilitan su enterramiento (Borrero 1988b).

Las dataciones señalan que han trascurrido al menos dos mil años para que sucedan situaciones de pisoteo por guanacos que impacten sobre el registro arqueológico. Entonces, cuando las condiciones para que esta acción ocurra son identificadas, su papel central es el de hipótesis nula, es decir, se debe demostrar que no afectó a los conjuntos bajo estudio. Ante condiciones habituales de interceptación tardía por parte de los arqueólogos de los conjuntos arqueológicos en médanos, donde los restos óseos muestran perfiles de meteorización avanzados, es difícil establecer la proporción entre estos y aquellos que pudieron ser depositados naturalmente. Es claro el problema para establecer conjuntos no ambiguos. El análisis contextual guiado por criterios explícitos como la presencia de modificaciones por procesamiento y consumo (huellas y golpes en primer lugar seguido por impacto térmico, más aún si se han identificado restos de carbón y/o fogones), junto con el contenido de nitrógeno en los restos, permiten establecer la existencia de un componente cultural en los conjuntos (L'Heureux y Borrero 2002).

Sobre la base de la información presentada se propone un continuum de "impronta antrópica" que se relaciona con el tiempo de exposición y el momento de interceptación de los conjuntos de artefactos y elementos faunísticos. En la escala del sitio cada contexto establece niveles de inferencia muy distintos, más aún respecto de los restos óseos. Desde la perspectiva distribucional, lo importante es que la señal arqueológica en términos de intensidad de uso del espacio en la escala regional puede ser identificada a la luz de un conjunto de distorsiones derivadas de las consideraciones tafonómicas. El contexto que presentaría menores perturbaciones en cuanto a contaminación de huesos más tardíos por acción del pisoteo y destrucción por meteorización de partes esqueletarias, es el "Nivel negro", seguido por el de superficie del mismo sitio "Huesos blancos" (sitio Santa Margarita 2) y el sitio Santa Margarita 1. La importante incidencia de la meteorización atenta contra la integridad de los restos, aunque se pudieron identificar elementos con evidencias de procesamiento / formatización (tal el caso de los restos datados y los instrumentos) a los que se suman los fragmentos quemados y calcinados. El caso extremo de dificultad de asignación cultural de restos está representado por el sitio Médano de Kaikén Aike 1. El gradiente conduce a pensar en conjuntos con material lítico pero cuya fauna se ha destruido. Esto es lo que parece haber sucedido en el caso de los muestreos finales de la transecta de Ea. San Agustín, donde se ven las mismas clases artefactuales que están representadas en los demás sitios (núcleos, desechos de talla, evidencias de manufactura de bolas, filos cortos y largos y manos y molinos). Dicha riqueza sugiere el desarrollo de un amplio rango de actividades, tal como se sugiere para los sitios Santa Margarita 2 y Médano de Kaikén Aike 1. Además, la redundancia en el uso humano de estos espacios sugiere su formación atricional, lo que eleva la expectativa de la utilización de fauna. Estos son los sitios en donde la inferencia acerca de la fauna "fantasma" tendría más sustento.

En términos metodológicos, una evaluación de la variabilidad del registro arqueológico en los médanos de la costa norte del Lago Viedma debiera focalizarse, en primer lugar, sobre los conjuntos líticos, y luego ponderar las situaciones de integridad de la fauna. No obstante, todos los efectos generados bajo las condiciones del Modelo de Pisoteo de guanaco 
en médanos también son relevantes para el estudio de los artefactos líticos (Borrazzo 2006; Borrazzo y Borrero 2015; Carranza 2017, 2018). En este sentido, se podría ampliar el alcance del modo tafonómico de médanos litorales propuesto por Borrazzo y Borrero (2015) a los médanos de los campos de invernada, y que así contenga al Modelo de Pisoteo.

El trabajo distribucional y la tafonomía regional han permitido identificar distorsiones sobre el registro arqueológico de la margen norte del Lago Viedma que se desprenden de reconocer las condiciones bajo las que opera el Modelo de Pisoteo y que ayudan a evaluar las formas de uso humano de la costa respecto de otros sectores ubicados en cotas más altas (pampas intermedias, mesetas). Este reconocimiento extiende la pertinencia del Modelo de Pisoteo (Borrero 1988a) más allá de Tierra del Fuego.
Agradecimientos: A Luis, por todo lo que seguimos aprendiendo.Y por su lectura y comentarios. A los Dres. María Gutiérrez, Gustavo Martínez y a los tres evaluadores anónimos por sus observaciones y sugerencias que fueron de gran ayuda para aclarar distintos aspectos del manuscrito. A la Sra. Ana Rojo (Ea. Punta del Lago), al Sr. Sergio Raggi (Ea. La Margarita), al Sr. Christian Rivera y al personal de la Ea. Santa Margarita y al Sr. Carlos Marcú (Ea. San Agustín) por su hospitalidad y ayuda en el campo. Al Dr. Luis Horta por las discusiones paleoambientales y, junto con el Sr. Pablo Binagui, por su inestimable colaboración en el campo. Al Dr. Gustavo Barrientos por su ayuda con las calibraciones de las dataciones. Los trabajos se realizan con subsidios otorgados por la Universidad Nacional de la Patagonia Austral (UARG), Proyecto 29/A304-1, CONICET PIP$11220120100622 \mathrm{CO}$ (Res. 4316) y MINCyT - ASETUR.

\section{Referencias Citadas}

Behrensmeyer, A. 1978. Taphonomic and ecologic information from bone weathering. Paleobiology 4 (2):150-162.

Belardi, J.B. y L.A. Borrero 1999. El paisaje arqueológico de la margen norte del lago Argentino (Provincia de Santa Cruz). Praehistoria 3:35-64.

Belardi, J.B. y S. Caracotche 2005. Resultados arqueológicos del noreste del lago Viedma. Provincia de Santa Cruz. Actas del XIII Congreso Nacional de Arqueología Argentina Tomo 4:81-88. Córdoba.

Belardi, J.B. y D. Rindel 2008. Taphonomic and archaeological aspect of massive mortality processes in guanaco (Lama guanicoe) caused by winter stress in Southern Patagonia. Quaternary International 180:38.51.

Belardi, J. B. D. Rindel y T. J. Bourlot 2012. Much more than it was expected: preservational differences of diaphysis and epiphyseal ends of guanaco (Lama guanicoe) long bones in Southern Patagonia (Argentina). Journal of Taphonomy 10 (1):45-65.

Belardi, J.B., G. Cassiodoro, R.A. Goñi, M. Glascock y A. Súnico 2015. Siltstone from Southern Patagonia: Its source and Archaeological Artifact Distribution in Santa Cruz Province, Argentina. Geoarchaeology: An International Journal 30:223-237.

Belardi, J.B., S. Espinosa, F. Carballo Marina, G. Barrientos, P. Campan y A. Súnico 2016. Desde la meseta del Cardiel Chico a la margen norte del lago Viedma (provincia de Santa Cruz, Argentina): nuevos datos sobre el paisaje arqueológico. En Arqueología de Patagonia: de Mar a Mar, editado por F. Mena, pp. 411-420. Ñire Negro Ediciones, CIEP, Coyhaique.

Belardi, J.B., F. Carballo Marina, P. Madrid, G. Barrientos y P. Campan 2017. Late Holocene guanaco hunting grounds in southern Patagonia: blinds, tactics and differential landscape use. Antiquity 91 (357):718-731.

Belardi, J.B, F. Carballo Marina, L.A. Borrero y D. Grima 2019a. Disponibilidad de campos de invernada e intensidad de uso del espacio en cuencas lacustres del Sur de Patagonia (Santa Cruz).
En Arqueología de la Patagonia: el Pasado en las Arenas, editado por J. Gómez Otero, A. Svoboda y A. Banegas, pp. 251-258. CONICET-IDEAUS, Buenos Aires.

Belardi, J. B, F. Carballo Marina, G. Barrientos y P. Campan. 2019b. Punta del Lago 1: implicaciones para la arqueología de la margen Norte del lago Viedma (Santa Cruz). En Arqueología de la Patagonia: El Pasado en las Arenas, editado por J. Gómez Otero, A. Svoboda y A. Banegas, pp. 351-359. CONICETIDEAUS, Buenos Aires.

Belardi, J.B, F. Carballo Marina y G. Barrientos 2021. Hunting landscapes in the north margin of Lake Viedma (Southern Patagonia, Argentina): Preys, strategies and technology. En Ancient Hunting Strategies in Southern South America, editado por J.B. Belardi, D. Bozzuto, P. Marcelo Fernández, E. Moreno y G. Neme, pp. 343-360. The Latin American Studies Book Series, Springer International Publishing, Cham.

Belardi, J.B, S.L, Espinosa, F. Carballo Marina y R.V. Blanco 2020. Al pie del cerro Fitz Roy: cazadores en el noroeste del lago Viedma, provincia de Santa Cruz. Revista del Museo de Antropología 13 (3):295-306.

Binford, L. 1987. Searching for camps and missing the evidence? Another look at the Lower Paleolithic. En The Pleistocene Old World. Regional Perspective, editado por O. Soffer, pp.17-30. Plenum Press, New York.

Binford, L. 1989. Debating Archaeology. Studies in Archaeology. Academic Press. San Diego

Borrazzo, K. 2006. Tafonomía lítica en dunas: una propuesta para el análisis de los artefactos líticos. Intersecciones en Antropología 7:247-261.

Borrazzo, K. y L.A. Borrero 2015. Taphonomic and archaeological perspectives from northern Tierra del Fuego, Argentina. Quaternary International 373:96-103.

Borrero, L.A. 1988a. Tafonomía Regional. En De Procesos, Contextos y otros Huesos, editado por N.R. Ratto y A.F. Haber, 
pp. 9-15. Facultad de Filosofía y Letras Universidad de Buenos Aires, Buenos Aires.

Borrero, L.A. 1988b. Estudios tafonómicos en Tierra del Fuego: su relevancia para entender procesos de formación del registro arqueológico. En Arqueología Contemporánea Argentina. Actualidad y Perspectivas, editado por H. Yacobaccio, pp. 13-32. Ediciones Búsqueda, Buenos Aires.

Borrero, L.A. 1989. Sites in action: the meaning of guanaco bones in Fueguian Archaeological sites. Archaeozoologia 3:9-24.

Borrero, L.A. 1989-90. Evolución divergente en la Patagonia Austral. Anales del Instituto de la Patagonia. Serie Ciencias Humanas 19:133-140.

Borrero, L.A. 1990. The Taphonomy of guanaco bones in Tierra del Fuego. Quaternary Research 34:361-371.

Borrero, L.A. 1993. Artefactos y evolución. Palimpsesto. Revista de Arqueología 3:15-32.

Borrero, L.A. 2001a. Regional taphonomy: the scales of application to the archaeological record. En Animals and Man in the Past. Essays in Honour of Dr. A. T. Clason Emeritus Professor of Archaeozoology Rijksuniversiteit Groningen, the Netherlands, editado por H. Buitenhuis y W. Prummel, pp. 17-20. ARCPublicatie 41. Groningen.

Borrero, L.A. 2001b. Regional taphonomy: Background noise and the integrity of the archaeological record. En Ethnoarchaeology of Andean South America. Contributions to Archaeological Method and Theory, editado por L. Kuznar, pp. 243.254. International Monographs in Prehistory, Ann Abor.

Borrero, L.A. 2009. Charles Darwin y la tafonomía. En 150 Años Después... la Vigencia de la Teoría Evolucionista de Charles Darwin, editado por M.C. Barboza, J.D. Avila, C. Piccoli y J. Cornaglia Fernández, pp. 107-121. Universidad Nacional Rosario, Rosario.

Borrero, L.A., J.L. Lanata y B.N. Ventura 1992. Distribuciones de hallazgos aislados en Piedra del Aguila. En Análisis Espacial en la Arqueología Patagónica, compilado por L.A. Borrero y J.L. Lanata, pp. 9-20. Ediciones Ayllu, Buenos Aires.

Carranza, E. 2017. Análisis tafonómico de conjuntos líticos de superficie en la costa norte del Golfo San Matías (Río Negro, Argentina). Intersecciones en Antropología 18 (1):91-101.

Carranza, E. 2018. The study of lithic assemblages on deflaction surfaces: The case study of Arroyo Verde, Northern Patagonia coast, Argentina. Journal of Lithic Studies 5 (2):DOI: https:// doi.org/10.2218/j1s.3030.

Cruz, I. y A.S. Muñoz 2010. Tafonomía comparativa: seguimiento de carcasas de mamíferos en Punta Entrada (Santa Cruz, Argentina). En Zooarqueología a Principios del Siglo XXI: Aportes Teóricos, Metodológicos y Casos de Estudio, editado por M.A. Gutiérrez, M. De Nigris, P.M. Fernández, M. Giardina, A.F. Gil, A. Izeta, G. Neme y H.D. Yacobaccio, pp. 387-396. Ediciones del Espinillo, Buenos Aires.

De Nigris M.E. y G.L. Mengoni Goñalons 2004. El guanaco como fuente de carne y grasas en Patagonia. En Contra Viento y Marea. Arqueología de Patagonia, editado por T. Civalero, P. Fernández y G. Guraieb, pp.469-476. Instituto Nacional de Antropología y Pensamiento Latinoamericano, Buenos Aires.
Dunnell, R. y W.S. Dancey 1983. The siteless survey: a regional scale data collection strategy. Advances in Archaeological Method and Theory 6:267-287.

Elkin, D. 1995. Volume density of South America camelids skeletal parts. International Journal of Osteoarchaeology 5:29-37.

Espinosa, S.L. y R.A. Goñi 1999. ¡ Viven!: una fuente de obsidiana en la provincia de Santa Cruz. En Soplando en el viento... Actas de las Terceras Jornadas de Arqueología de la Patagonia, pp. 177-188. Instituto Nacional de Antropología y Pensamiento Latinoamericano y Universidad Nacional del Comahue, Neuquén.

Foley, R. 1981. Off-Site Archaeology and Human Adaptation in Eastern Africa. An Analysis of Regional Artefact Density in the Amboseli, Southern Kenia. Cambridge Monographs in African Archaeology 3. BAR International Series 97, Oxford.

Hajduk, A. y M.J. Lezcano 2005. Un "nuevo-viejo" integrante del elenco de instrumentos óseos de Patagonia: los machacadores óseos. Magallania 33 (1):63-80.

Kaplan, M.R., J.M. Schaefer, J.A. Strelin, G.H. Denton, R.F. Anderson, M.J. Vandergoes, R.C. Finke, R. Schwartz, S.G. Travis, J.L. García, M.A. Martini y S.H.H. Nielsen 2016. Patagonian and southern South Atlantic view of Holocene Climate. Quaternary Science Reviews 141:112-125.

Laland, K.N. y M.J. O'Brien 2010. Niche construction theory and archaeology. Journal of Archaeological Method and Theory 17:303-322.

L'Heureux, G.L. y L.A. Borrero 2002. Pautas para el reconocimiento de conjuntos óseos antrópicos y no antrópicos de guanacos en Patagonia. Intersecciones en Antropología 3:29-40.

Menghin, O. 1957. Estilos del arte rupestre de Patagonia. Acta Praehistórica 1:57-82.

Muñoz, A.S. 1999. El registro arqueofaunístico del sitio Campo del Lago 2. Implicaciones para el estudio de los procesos de formación del registro arqueológico en la costa sur del lago Argentino (Provincia de Santa Cruz, Argentina). Praehistoria 3:105-117.

Nuevo Delaunay, A., J.B. Belardi y F. Carballo Marina 2020. Nuevas evidencias de sitios arqueológicos Tehuelche/AonikenkMapuche (siglo XX) en Santa Cruz, Patagonia (Argentina) Magallania 48 (1):161-172.

Pereyra, F.X., L. Fauqué y E.F. González Díaz 2002. Geomorfología. En Relatorio del XV Congreso Geológico Argentino. Geología y Recursos Naturales de Santa Cruz, editado por M. Haller, Parte I: Geología, pp. 325-352. Asociación Geológica Argentina, Buenos Aires.

Popper, K. 1980. La Lógica de la Investigación Científica. Editorial Tecnos, Madrid.

Rindel, D. y J.B. Belardi 2006. Mortandad catastrófica de guanaco por estrés invernal y sus implicaciones arqueológicas en el sitio Alero Los Guanacos 1 (lago Cardiel, provincia de Santa Cruz, Argentina) Magallania 34 (1):139-155.

Rossignol, J. y L.A. Wandsnider 1992. Space, Time and Archaeological Landscapes. Plenum Press, New York.

Strelin, J. y E. Malagnino 1996. Glaciaciones pleistocénicas del lago Argentino y alto valle del río Santa Cruz. Actas XIII 
Congreso Geológico Argentino. III Congreso de Exploración de Hidrocarburos 4:311-325. Asociación Geológica Argentina, Instituto Argentino del Petróleo y del Gas, Buenos Aires.

Strelin, J.A., M.R. Kaplan, M.J. Vandergoes, G.H. Denton y J.M. Schaefer 2014. Holocene glacier history of the Lago Argentino basin, Southern Patagonian Icefield. Quaternary Science Reviews 101:124-1455.

Stuiver, M., P.J. Reimer y R.W. Reimer 2020. CALIB 8.2 [WWW program] available at disponible en http://calib.org

Sturzembaum, P. y P. Borrelli 2001. Manejo de riesgos climáticos. En Ganadería Ovina Sustentable en la Patagonia Austral.
Tecnologías de Manejo Extensivo, editado por P. Borrelli y G. Oliva, pp. 255-270. INTA y ErreGé, Santa Cruz y Buenos Aires.

Thomas, D.H. 1975. Nonsite sampling in archaeology. Up the Creek Without a site? En Sampling in Archaeology, editado por J.E Mueller, pp. 61-81. The University of Arizona Press, Tucson.

Winterhalder, B. y E.A. Smith 1992. Evolutionary ecology and the social sciences. En Evolutionary Ecology and Human Behavior, editado por E. A. Smith y B. Winterhalder, pp. 3-23, Aldine de Gruyter, New York.

Wenzens, G., E. Wenzens y G. Schellman 1997. Early Quaternary of glacial y eolian forms in semiarid Patagonia. Zeitschriftfür Geomorphology. NeueFolge. Supplementband 111:131-144. 
